\title{
Study on Effects of NHERF-1 on Proliferation and Migration of Human Umbilical Vein Endothelial Cells
}

\section{Li Dan ${ }^{1}$, Chu Xianming ${ }^{1}$, Feng Zhihui ${ }^{2}$, Li Xia ${ }^{1}$, Chen Peng ${ }^{3}$, and An Yi ${ }^{1}$}

${ }^{1}$ Department of Cardiology, The Affiliated Hospital of Qingdao University, Qingdao 266003, China

${ }^{2}$ Institute of Transfusion medicine, Qingdao Blood Center, Qingdao 266073, China,

${ }^{3}$ Qingdao University, Qingdao 266071, China

Correspondence should be addressed to

An Yi: 18661627280@163.com

\begin{abstract}
Object To explore the effects of $\mathrm{Na}^{+} / \mathrm{H}^{+}$exchanger regulatory factor 1 (NHERF1) on the phosphorylation levels of Akt1, activity of gelatinase secreted by HUVECs, and expression and distribution of cytoskeleton inside Human Umbilical Vein Endothelial Cells (HUVECs), and to expound the molecular mechanism of NHERF1 to influence the proliferation, migration and angiogenesis of vascular endothelial cells. Methods To construct the recombinant eukaryotic expression plasmid of NHERF1 and stably transfect HUVEC line with recombinant plasmid respectively; the 3-4,5-Dimethyl-2-thiazolyl-2,5-diphenyl-2H- tetrazolium bromide (MTT) was used to assay the proliferation activity of HUVECs after verification with the Western blotting method; to adopt the scarification test to measure the migration activity of HUVECs; to apply the Matrigel method to detect the angiogenesis ability of NHERF1 in cells; to detect the effect of NHERF1 on the phosphorylation levels of Akt1 by Western blotting; to use the gelatin zymography analysis to test the activity of gelatinase secreted by HUVECs; to observe the distribution of cytoskeleton with the immunofluorescence. Results The cDNA fragments of the exogenous NHERF-1 transfected have been integrated into the genome; NHERF-1 can significantly inhibit the proliferation, migration and angiogenesis of HUVECs, obviously down-regulate the phosphorylation levels of Akt11, make HUVECs decrease the secretion of reduction proenzyme and active enzyme, and also influence the distribution of cytoskeleton in vascular endothelial cells, compared to the cells in the control group. Conclusion NHERF1 can inhibit proliferation, migration and angiogenesis of HUVECs, and the inhibiting mechanism might relate to adjustment of the phosphorylation levels of Akt11, regulation of the activity of gelatinase secreted by HUVECs, and influence on the distribution of microfilament cytoskeleton.
\end{abstract}

Keywords: NHERF1, Vascular endothelial cells, Proliferation, Migration

\section{Introduction}

Angiogenesis refers to the process that new blood vessels grow from the existing blood vessels, and plays an important role in cell replication, wound healing, inflammatory response, and other processes. This process is strictly regulated and controlled, and any violation of the regulation mechanism may cause severe pathological changes, including rheumatic arthritis, psoriasis, and malignancy. Therefore, the abnormally active angiogenesis is an important condition of malignancy growth. Early in 1971, Folkman ${ }^{[1]}$ proposed that malignancy growth was vessel-dependent and 
divided such growth process into two phases, i.e. non-vascular phase and vascular one. Vascularized tumor growth may accelerate and easily have metastasis. Tumor angiogenesis is a complex pathophysiological process, including proteases release by endothelial cells, matrix membrane degradation, migration and proliferation of endothelial cells, and lumen formation of endothelium cells. This process is regulated and controlled by various angiogenic factors (angiogenesis-promoting factor and angiogenesis-inhibitory factor) and related genes ${ }^{[2]}$. Inhibiting the formation of new vessels has become a new strategy for tumor treatment.

However, angiogenesis is a complex network regulation and control process, while the network balance between pro-angiogenic and anti-angiogenic factors has been proved to play a key role in different links. Therefore, the major direction of future research should focus on explaining the regulatory network of corneal neovascularization in different depths and breadths, seeking specific control points with key functions and further developing effective, safe and economic therapeutic plans.

There is no blood vessel in cornea itself, so any vessel found in the experiment can be treated as a new blood vessel. In this way, the interference of original vessels in other models can be avoided so as to truly reflect the process of angiogenesis ${ }^{[3]}$. In 1974, Folkman et al. applied this model to the research on tumor angiogenesis for the first time. In the early studies on tumor angiogenesis, a corneal neovascularization model of mice induced by suture for 5 days was established successfully. The expression of NHERF-1 (Na+/H+exchanger regulatory factor 1) showed significant up-regulation in the process of neovascularization according to the differential expression genes screened with gene expression profile chips in angiogenesis.

Widely distributing in tissues, NHERF1 is a protein containing 358 amino acids. Composed of two serial PDZ structural domains (PDZ-1 and PDZ-2) at the amino terminal and ERM structural domain at the carboxyl terminal, it is highly expressed in mammary gland, liver, bile duct, pancreas and salivary gland. A lot of studies on the protein-protein interaction among these three structural domains showed that NHERF1 can combine more than 30 proteins, including SYK ${ }^{[4]}$, PTEN ${ }^{[5]}$, $\operatorname{PDGFR}^{[5-7]}$, PLC $\beta, \beta$-catenin, ERM family proteins ${ }^{[8,9]}$, and etc. to regulate and integrate various signaling pathways.

The existing research results showed that NHERF1 plays a role in regulation of ion transporters, transportation and stabilization of transmembrane protein, and composition of apical microvillus structure in epithelium. In recent years, the studies on NHERF1 mainly focused on its ability to inhibit cancer, which may be realized by interaction with various cancer suppressor proteins (for example, phosphorylated tumor suppressor genes, moesin, tyrosine kinase, and etc.), enhancement of stability of tumor suppressor protein, and inhibition of carcinogenetic function by combining carcinogenetic proteins $^{[4,8,9]}$. At present, no report on the role and mechanism of NHERF1 in tumor angiogenesis is available yet. In this study, the effects of NHERF-1 protein on the proliferation, migration and angiogenesis functions of human vascular endothelial cells and its possible molecular mechanism were researched by excessively expressing NHERF1 protein in HUVECs.

\section{Materials and methods}

\section{Materials}

HUVEC kindly sent by Cora-Jean S. Edgell from University of North Carolina, DMEM dry powder (Invitrogen Corporation, U.S.), penicillin sodium for injection (Harbin General Pharmaceutical Factory, China), streptomycin sulphate for injection (Shandong Lukang Pharmaceutical Co., Ltd., China), trypsin (Gibco Inc., U.S.), VN mouse anti-human monoclonal antibody (Santa Cruz Biotechnology Inc., U.S.), goat anti-mouse IgG antibody marked by horse 
radish peroxidase (Beijing Zhongshan Golden Bridge Biotechnology Co., Ltd., China), mouse anti-GAP-DH antibody (Shanghai KangChen Bio-tech, China), Western Blot luminescence reagent (Thermo Fisher Scientific, U.S.), FITC-Phalloidin (phalloidin) (Sigma-Aldrich, U.S.), P-Akt11 anti-body and HA antibody (Cell Signaling Technology Inc. U.S.), and Akt11 antibody (Abcam, U.S.).

Construction of eukaryotic expression vector

RNA was extracted from HUVECs and reversely transcribed into cDNA. The following primer was used to amplify the full-length sequence of NHERF1, and EcoRI and XhoI enzyme cutting sites were introduced into the both ends of the amplified sequence: forward: AGAGAATTCAGCGCGGACGCAGCGG; and reverse: CCGCTCGAGTCAGAGGTTGCTGAAGAGT. Then, the expression vector pBK-CMV-HA and full-length sequence of amplified NHERF1 were cut as cohesive terminus with EcoRI and XhoI enzyme. Cutting gel was recycled after gel electrophoresis to connect and transform escherichia coli DH5 $\alpha$ for resistance screening. After positive clones were obtained, plasmid was extracted for sequencing. It was confirmed that the obtained pBK-CMV-HA-NHERF1 positive clones had correct sequence and reading frame.

Establishment and identification of stably transfected cell line

HUVECs were cultured in an incubator with $5 \% \mathrm{CO}_{2}$ at $37{ }^{\circ} \mathrm{C}$ in the medium containing DMEM-F12 with 10\% fetal bovine serum and 1\% penicillin and streptomycin (Dulbecco's Modified Eagle Medium/F12). HUVECs were transfected by $2 \mu \mathrm{g}$ recombined plasmid DNA through mediating with liposome (Hifectin II). Resistant cell clone was obtained after screening with the selective medium in a concentration of $400 \mu \mathrm{g} / \mathrm{m} 1 \mathrm{G} 418$ (Geneticin, aminoglycoside of gentamicin) for 3-4 weeks. After digestion and separation, culture and passage were conducted with the maintenance medium of G418 $(200 \mu \mathrm{g} / \mathrm{ml})$. The HUVEC line stably transfecting pBK-CMV-HA-NHERF1 was named as NHERF1-HUVEC, and that stably transfecting pBK-CMV-HA as HA-HUVEC. The two cells were inoculated in the $60 \mathrm{~mm}$ cell culture dishes, respectively. After the cells adhered to the wall and the density grew to about $80 \%$, total cellular protein was extracted, respectively. The content of interest protein in cells was measured with the Western blot method as follows: the expression level of exogenously transferred NHERF1 protein was detected by HA antibody, and the total expression levels of endogenic and exogenous NHERF1 protein in transfected and non-transfected cells were detected by NHERF1 antibody. Then, positive clone with the stable and excessive expression of exogenous NHERF1 was obtained by scanning and analyzing the results of the Western blot experiment with the Image $\mathrm{J}$ image analysis system.

Cell proliferation detected with the MTT method

Two kinds of HUVECs after stable transfection were digested into cell suspension, respectively. Then, the cells were inoculated in 96 -well plate in a concentration of $1 \times 103$ cells $200 \mu 1 /$ well and cultured at $37^{\circ} \mathrm{C}$. Three duplicated wells were selected from each group of cells every 24 hours, and $20 \mathrm{ug}$ of MTT was added to each of them for continuous 4-hour culture; then the nutrient solution was absorbed before $100 \mathrm{ul}$ of DMSO was added to measure absorbance at $492 \mathrm{~nm}$, at $37^{\circ} \mathrm{C}$, for 10 minutes.

Cell migration detected by scarification

Two kinds of HUVECs after stable transfection were inoculated in the $30 \mathrm{~mm}$ culture dish, respectively. After the cell density increased to $100 \%$, two groups of cells were scarified by $100 \mathrm{ul}$ micro pipette tip with the width of about 500um. After 2 times of PBS washing, the cells were added to serum-free mediums and cultured in an incubator for 16 hours, respectively. After scarifying and 
photographing 2 groups of cells, 10 views were selected randomly to calculate the quantity of the cells which have migrated into the scratched area.

Angiogenesis capability of cells detected by the Matrigel assay

Matrigel was placed in a refrigerator at $4^{\circ} \mathrm{C}$ for 2 hours to dissolve it completely. Then, Matrigel was added to the pre-cooled 48 -well plate with $150 \mathrm{ul}$ per well at $37^{\circ} \mathrm{C}$ for 1 hour so as to form gel. Two kinds of HUVECs after stable transfection were digested into cell suspension, respectively, and incubated in the 18 -well plate with gel laying in the density of $70 \times 10^{6}$ cells/L. With $200 \mu 1$ suspension in each well, the plate was photographed after being cultured in an incubator for 12 hours. Then, 6 views were selected randomly to observe and calculate the quantity of the vessel lumens.

Western blot

Two kinds of HUVECs after stable transfection were inoculated in the $60 \mathrm{~mm}$ culture dish, respectively. After the cell density grew to $80 \%$, total protein was extracted from each group of cells. Then, $20 \mu 1$ of cell lysis buffer was taken from each group and transferred to nitrocellulose membrane through $8 \%$ SDS-PAGE for 1-hour sealing. After incubation at $4{ }^{\circ} \mathrm{C}$ overnight, the primary antibody was coupled with the secondary antibody with horse radish peroxidase for 1 hour of reaction at room temperature. Then, coloration was conducted with the ECL method. The result of the Western blot experiment was scanned and analyzed with the Image $\mathrm{J}$ image analysis system.

Gelatin zymography assay

The activity of MMP-2 and MMP-9 was detected with $8 \%$ SDS-PAGE containing $0.1 \%$ gelatin. $20 \mu \mathrm{l}$ of the nutrient solution after 48-hour serum-free culture was taken from each group for electrophoresis at a $120 \mathrm{~V}$ constant voltage for $2 \sim 2.5$ hours. After the gel was rinsed with $2.5 \%$ TritonX-100 for three times, the reaction buffer $(\mathrm{pH} 7.6,50 \mathrm{mmol} / \mathrm{L}$ Tris-HCL, $10 \mathrm{mmol} / \mathrm{L} \mathrm{CaCl}$, $50 \mathrm{mmol} / \mathrm{L} \mathrm{NaCl}$, and $0.05 \%$ Brij35) was added to incubate at $37^{\circ} \mathrm{C}$ for 17 hours. Then, staining was conducted with $0.5 \%$ Coomassie brilliant blue for $1.5 \sim 2$ hours, and decoloration was conducted with the destainer $(10 \%$ acetic acid-40\% methyl alcohol) until white strip was clear on the blue background. The experiment result was scanned and analyzed with the Image $J$ image analysis system.

Changes in cell microfilament cytoskeleton (F-actin) observed with the immunofluorescence method

Fluorescent staining was directly conducted upon the microfilament with the phalloidine fluorescence probe (FITC-Phalloidin). The specific steps are as follows: Two kinds of HUCECs after stable transfection were inoculated in a culture dish with glass bottom until the density was up to $50 \%$, respectively. Then, the cells were washed with PBS for three times. The cells were fixed with $4 \%$ neutral paraformaldehyde at room temperature for $15 \mathrm{~min}$, and then washed with PBS for three times. The cells were processed with $0.1 \%$ Triton X-100 for $5 \mathrm{~min}$, and then washed with PBS for three times. FITC-Phalloidin diluted by 1:200 was added dropwise. Then, the cells were kept out of light at room temperature in a wet box for 30min and washed with PBS for three times. The cell climbing sheet after staining was observed under the confocal laser scanning microscope (Eclipse TE2000-U, Nikon, Tokyo, Japan).

Statistical analysis

The SPSS13.0 statistic software was used to conduct statistical analysis on the data. All the experiment data were expressed as mean \pm standard deviation, and all the experiments were repeated for 3 times. A homogeneity test of variances was conducted to each sample firstly. Then, the growth curve measurement was analyzed by repeated measurement in $T$-test, while the data of other experiments were compared with the $t$-test of independent samples. $\mathrm{P}<0.05$ meant that the difference was statistically significant. 


\section{Results}

Expression of endogenous NHERF-1 in HUVECs

The expression of endogenous HHERF-1 in HUVECs was detected by RT-PCR as shown in Fig. 1.

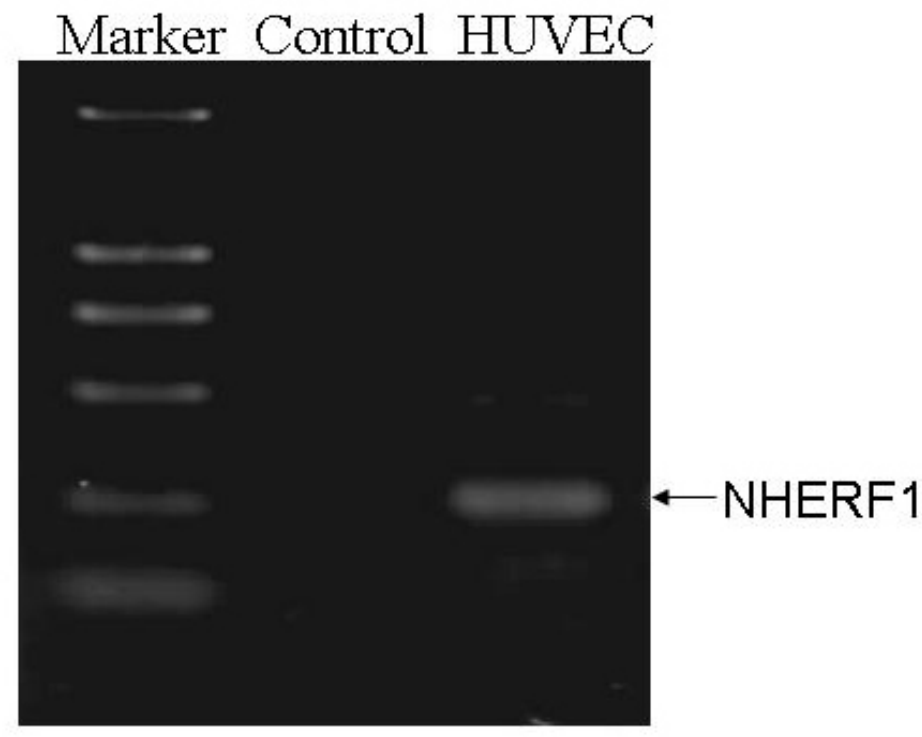

Fig.1. The expression of endogenous NHERF1 in HUVECs detected by RT-PCR

Identification of stably transfected cell line

In order to obtain the positive clone with stable and excessive expression of exogenous NHERF1, the content of interest proteins were tested in the two groups of HUVECs transfecting pBK-CMV-HA-NHERF1 and pBK-CMV-HA as shown in Fig. 2.

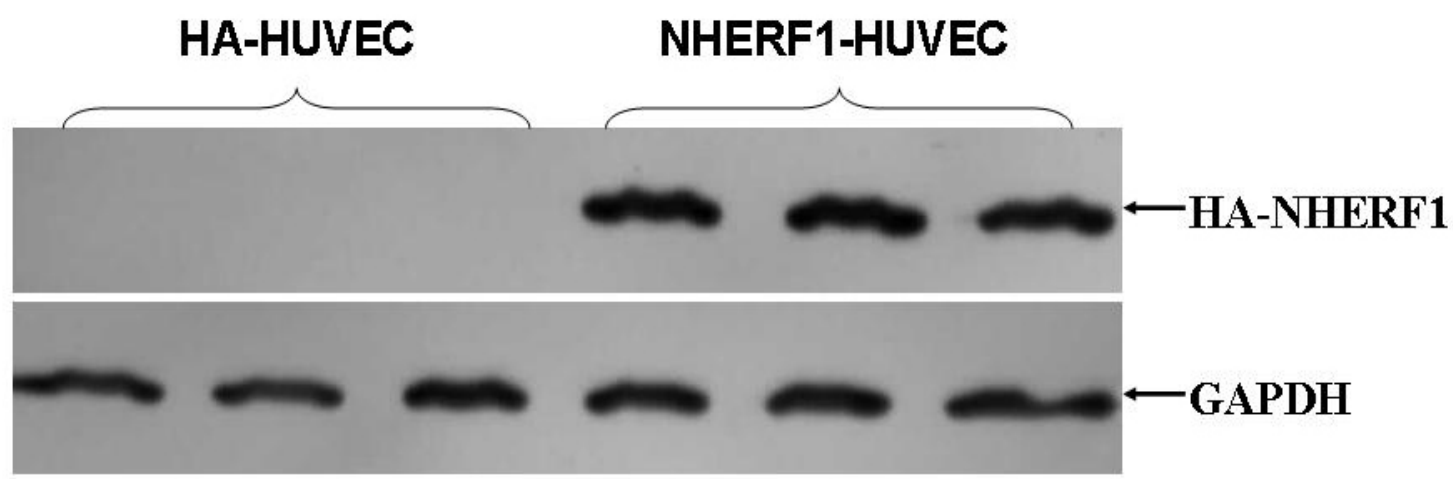

Fig. 2 Identification of HUVEC lines stably expressing NHERF1

Effect of NHERF1 on the proliferation of HUVECs

The proliferation capacity of HA-HUVECs and NHERF1-HUVECs was detected with the MTT method. The result showed that the proliferation capability of NHERF1-HUVECs with excessive expression of NHERF1 protein decreased significantly when compared with HA-HUVECs in the control group $(\mathrm{P}=0.02)$ as shown in Fig. 3. 


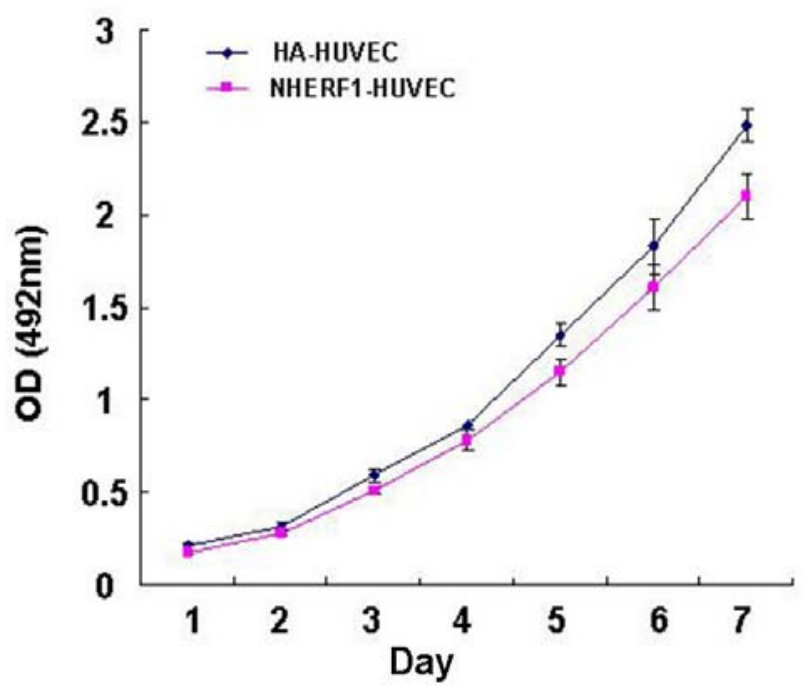

Fig. 3. Effect of NHERF1 on the proliferation of HUVECs, $\mathrm{P}=0.02$.

Effect of NHERF1 on the migration of HUVECs

The migration capability of HA-HUVECs and NHERF1-HUVECs was detected by scarification as shown in Fig. 4A. The statistical result showed that the migration capability of NHERF1-HUVECs with excessive expression of NHERF1 protein decreased significantly by about $20 \%$ when compared with HA-HUVECs in the control group $(\mathrm{P}=0.02)$ as shown in Fig. 4B.

A

\section{HA-HUVEC}

$\mathrm{OH}$

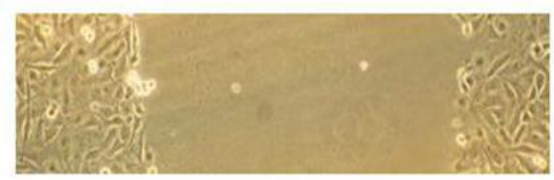

$16 \mathrm{H}$

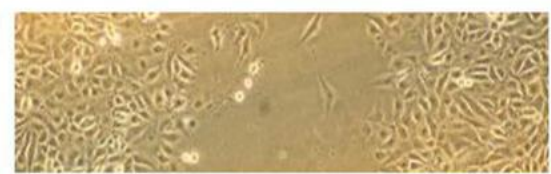

NHERF1-HUVEC
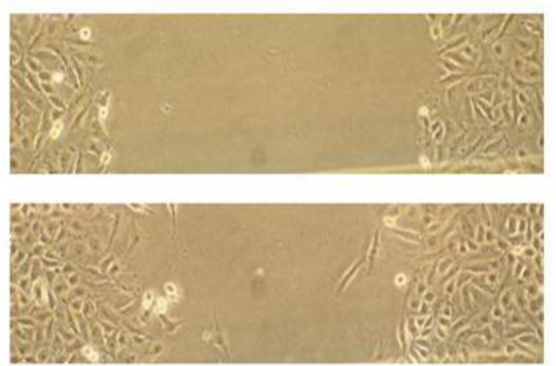

B

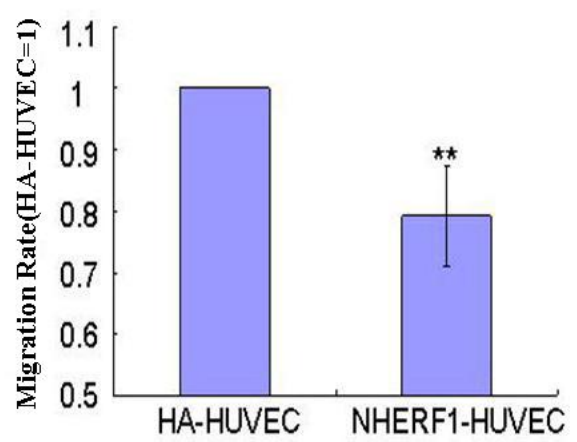

Fig. 4. Effect of NHERF1 on the migration of HUVECs, $\mathrm{P}<0.01$

Effect of NHERF1 on the angiogenesis of HUVECs

The angiogenesis capability of HA-HUVECs and NHERF1-HUVECs was detected with the Matrigel method. The result showed that the lumen formation quantity and length of NHERF1-HUVECs with excessive expression of NHERF1 protein decreased significantly when compared with HA-HUVECs in the control group as shown in Fig. 5. 


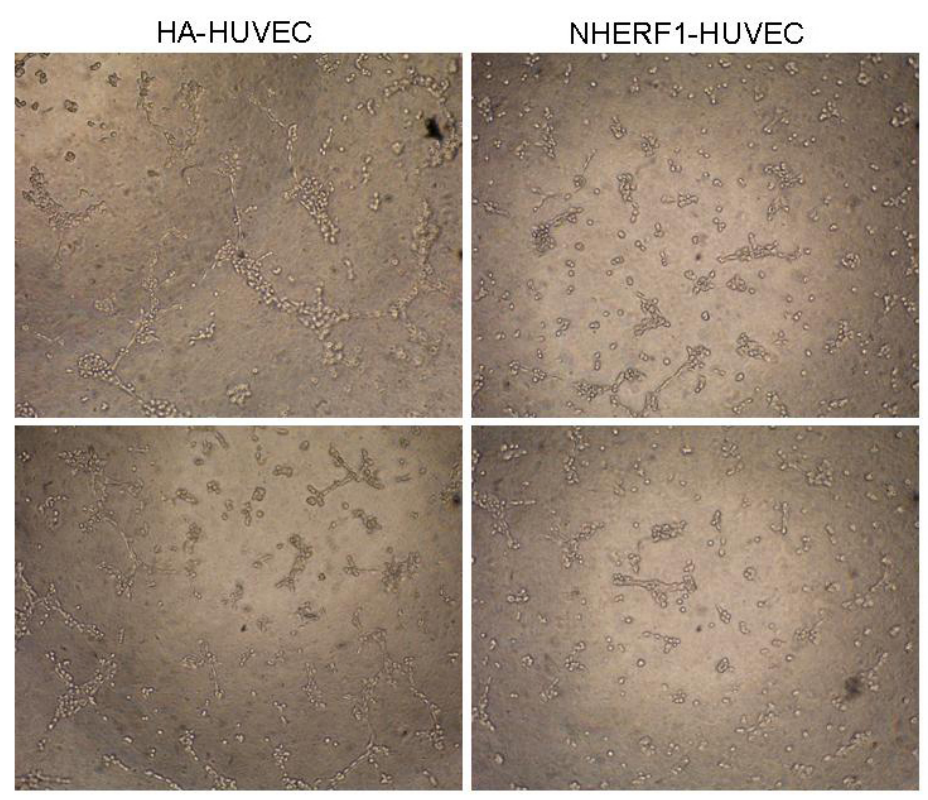

Fig. 5. Effect of NHERF1 on the angiogenesis capability of HUVECs

Effect of NHERF-1 expression on the Akt11 phosphorylation levels of HUVECs: The Akt11 phosphorylation levels in HA-HUVECs and NHERF1-HUVECs were detected by Western blot as shown in Fig. 6A. The statistical result showed that the Akt11 phosphorylation levels of NHERF1-HUVECs with excessive expression of NHERF1 protein decreased significantly by about $40 \%$ when compared with HA-HUVECs in the control group as shown in Fig. 6B.
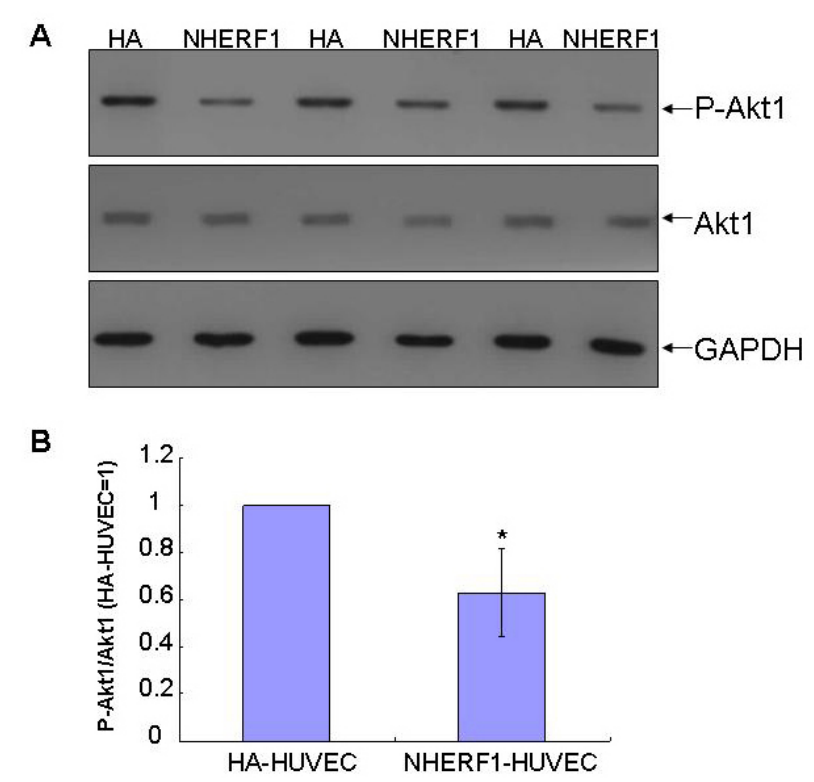

Fig. 6. NHERF-1 expression decreased the Akt11 phosphorylation level in HUVECs

Effect of NHERF-1 expression on the activity of gelatinase secreted by HUVECs

The activity of gelatinase in HA-HUVECs and NHERF1-HUVECs was detected by gelatin zymography as shown in Fig. 7. Compared with HA-HUVECs in the control group, the activity of MMP2 in NHERF1-HUVECs with excessive expression of NHERF1 protein decreased by about $50 \%(\mathrm{P}<0.01)$, while the activity of MMP-9 showed no significant change. 


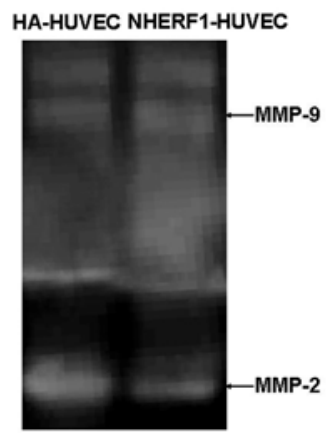

Fig. 7 Effect of NHERF-1 expression on the activity of gelatinase secreted by HUVECs

Effect of NHERF-1 expression on the distribution of cell microfilament cytoskeleton

The distribution of cell microfilament cytoskeleton in HA-HUVECs and NHERF1-HUVECs was detected with the immunocytochemical method as shown in Fig. 8. There was no significant difference in the fluorescent intensity between HUVECs transfected by NHERF-1 and NHERF1-HUVECs by unloading transfection, and both kinds of cells widely distributed in cytoplasm. It was easy to see that F-actin in HUVECs transfected with NHERF-1 was filamentous. The fiber arranged in parallel with even thickness and distributed along the cell polarity.
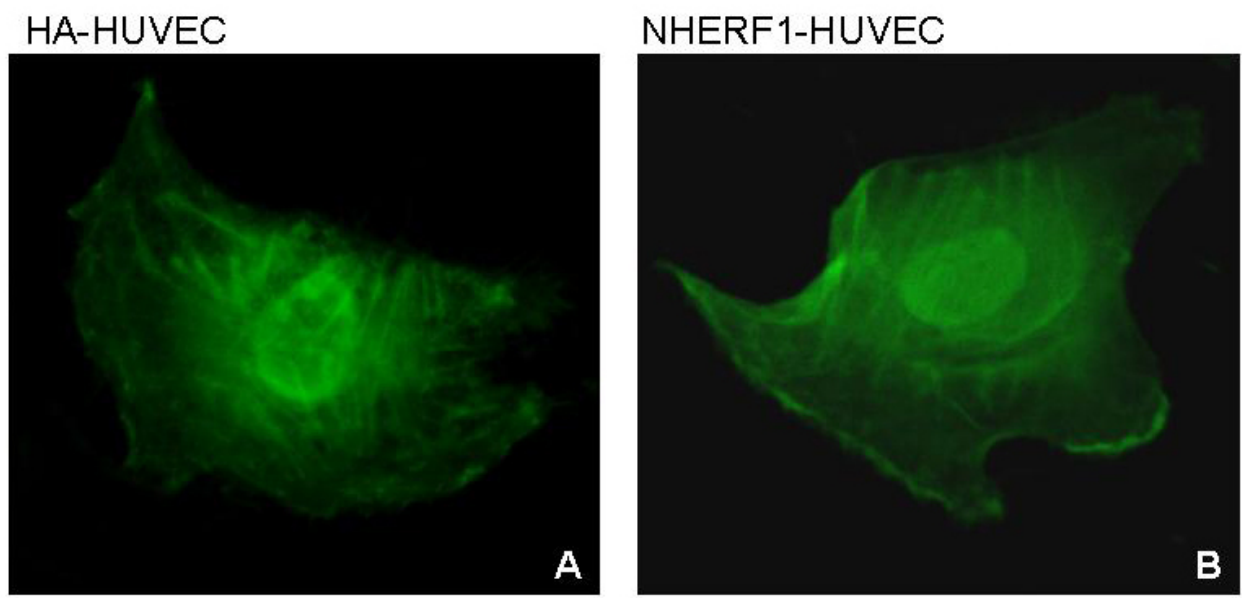

Fig. 8. Effect of NHERF-1 expression on the distribution of cell microfilament cytoskeleton in HUVECs

\section{Discussion}

As a multi-functional ligandin with the potential of inhibiting cancer found recently ${ }^{[10]}$, NHERF1 plays a very important role in cell growth, chemotaxis and migration ${ }^{[5-7,9,11-15]}$. Simoncini $\mathrm{T}$ et al. ${ }^{[16]}$ have detected the expression of NHERF1 in HUVECs and speculated that it might play a certain role in angiogenesis. Song GJ et al. ${ }^{[17]}$ found that NHERF1 is only expressed in normal vascular endothelial cells, but almost not in vascular smooth muscle cells. After damage, however, the expression of NHERF1 significantly increased in vascular smooth muscle cells. Particularly, the expression of NHERF1 in cells with new endangium significantly increased in the process of angiogenesis. All of these studies showed that NHERF1 may play an important role in angiogenesis.

The results of early studies have shown that NHERF1 is one of genes with significant up-regulation of expression in angiogenesis. Up to now, it has been found that only estrogen can activate the NHERF1 expression in cells. However, further studies are still required on the role and 
mechanism of NHERF1 in angiogenesis. We have found from the research that NHERF1 can significantly inhibit the proliferation, migration, and angiogenesis capability of HUVECs.

Phosphatidylinositol 3-kinase (PI3Ks) signals participate in the adjustment of various cellular functions, including proliferation, differentiation, apoptosis, and glucose transport. In recent years, the signal path composed of IA-type PI3K and other downstream molecular protein kinase B (PKB or Akt1) is closely related to the growth and development of human cancers. This path can adjust the proliferation and survival of cancer cells. Its activity abnormality may not only cause malignant transformation of cells, but also be related to the migration, adherence, tumor angiogenesis, and degradation of extracellular matrix ${ }^{[18-24]}$. Nowadays, the treatment strategy targeting at key molecules on the PI3K-Akt1 signal path is being developed. Some of the current studies showed that in certain cancer cells, NHERF1 plays a role of negative regulation over the Akt11 signal path.

The MMP family is closely related to cells' migration and proliferation ${ }^{[25-27]}$. As one of the MMP family members, Gelatinase includes gelatinase A (MMP-2) and gelatinase B (MMP-9). Both kinds of gelatinase are secreted in the form of zymogen and have the molecular weight of $72 \mathrm{kD}$ and $92 \mathrm{kD}$ (or $95 \mathrm{kD}$ ), respectively. After activation, the molecular weight of MMP-2 may be $68 \mathrm{kD}, 66 \mathrm{kD}$ or $62 \mathrm{kD}$, while that of MMP-9 may be $88 \mathrm{kD}$ or $85 \mathrm{kD}$. The substrate of gelatinase includes gelatin, collagen IV, V, VII, and X, as well as fibronectin. In vivo, gelatinase can degrade collagen IV, one of the major structural components of basilar membrane, playing a crucial role in the penetration and metastasis of cancer cells through extracellular matrix $(\mathrm{ECM})^{[28,29]}$. Therefore, this gelatinase is closely related to the growth and development of cancer.

Cytoskeleton is a network system composed of intracellular protein, including microtubule, microfilament, and intermediate filament. As the major structure regulating cell adherence, extension and morphology, microfilament plays an important role in multiple functions of cells, including proliferation. Moreover, microfilament is also the member with the closest relationship with the apoptosis signal molecules in cytoskeleton. Microfilament cytoskeleton may recombine in cellular differentiation and movement, and play a role of mediating extracellular signal molecules ${ }^{[30-32][4,6,7]}$. Phalloidin can specifically bind F-actin, the major component of stress fiber in microfilament cytoskeleton, so the dynamic changes of F-actin in cells can be observed clearly under a laser scanning confocal microscope ${ }^{[33]}$.

In order to further study the mechanism of NHERF1 in significant inhibition of proliferation and migration of HUVECs, we detected the effects of NHERF1 on the Akt1 signal path, the activity of collagenase IV and microfilament cytoskeleton, and the results showed that NHERF1 can significantly down-regulate the phosphorylation levels of Akt1, reduce gelatinase and active enzyme secreted by HUVECs, and change the microfilament structure in cells. In conclusion, NHERF-1 can inhibit the proliferation and migration of HUVECs, and this may be related to its regulation over the phosphorylation levels of Akt1, control over the activity of gelatinase secreted by cells and the effect on the distribution of microfilament cytoskeleton.

\section{References}

[1] Folkman J. Tumor angiogenesis: therapeutic implications. N Engl J Med, 1971, 285:1182-1186.

[2] Carmeliet P, Jain RK. Angiogenesis in cancer and other diseases. Nature, 2000, 407:249-257.

[3] Auerbach R, Lewis R, Shinners B, et al. Angiogenesis assays: a critical overview. Clin Chem, 2003,49:32-40. 
[4] Dai JL, Wang L, Sahin AA, et al. $\operatorname{NHERF}\left(\mathrm{Na}^{+} / \mathrm{H}^{+}\right.$exchanger regulatory factor) gene mutations in human breast cancer. Oncogene,2004, 23:8681-8687.

[5] Takahashi Y, Morales FC, Kreimann EL, et al. PTEN tumor suppressor associates with NHERF proteins to attenuate PDGF receptor signaling. EMBO J 2006,25:910-920.

[6] Maudsley S, Zamah AM, Rahman N, et al. Platelet-derived growth factor receptor association with $\mathrm{Na}^{+} / \mathrm{H}^{+}$exchanger regulatory factor potentiates receptor activity. Mol Cell Biol, 2000, 20:8352-8363.

[7] James MF, Beauchamp RL, Manchanda N, et al. A NHERF binding site links the betaPDGFR to the cytoskeleton and regulates cell spreading and migration. J Cell Sci, 2004, 117:2951-2961.

[8] Murthy A, Gonzalez-Agosti C, Cordero E, et al. NHE-RF, a regulatory cofactor for $\mathrm{Na}^{+}-\mathrm{H}+$ exchange, is a common interactor for merlin and ERM (MERM) proteins. J Biol Chem, 1998, 273:1273-1276.

[9] Reczek D, Berryman M, Bretscher A. Identification of EBP50: A PDZ-containing phosphoprotein that associates with members of the ezrin-radixin-moesin family. J Cell Biol, 1997, 139:169-179.

[10] Georgescu MM, Morales FC, Molina JR, et al. Roles of NHERF1/EBP50 in cancer. Curr Mol Med, 2008, 8:459-468.

[11] Weinman EJ, Steplock D, Tate K, et al. Structure-function of recombinant $\mathrm{Na} / \mathrm{H}$ exchanger regulatory factor (NHE-RF). J Clin Invest,1998, 101:2199-2206.

[12]Ladias JA. Structural insights into the CFTR-NHERF interaction. J Membr Biol, 2003, 192:79-88.

[13] Cao TT, Deacon HW, Reczek D, et al. A kinase-regulated PDZ-domain interaction controls endocytic sorting of the beta2-adrenergic receptor. Nature, 1999, 401:286-290.

[14] Suh PG, Hwang JI, Ryu SH, et al. The roles of PDZ-containing proteins in PLC-beta-mediated signaling. Biochem Biophys Res Commun,2001, 288:1-7.

[15]Demoulin JB, Seo JK, Ekman S, et al. Ligand-induced recruitment of $\mathrm{Na}^{+} / \mathrm{H}^{+}$-exchanger regulatory factor to the PDGF (platelet-derived growth factor) receptor regulates actin cytoskeleton reorganization by PDGF. Biochem J. 2003. 376:505-510.

[16] Simoncini T, Scorticati C, Mannella P, et al. Estrogen receptor alpha interacts with Galpha13 to drive actin remodeling and endothelial cell migration via the RhoA/Rho kinase/moesin pathway. Mol Endocrinol. 2006. 20:1756-1771.

[17] Song GJ, Barrick S, Leslie KL, et al. EBP50 inhibits the anti-mitogenic action of the parathyroid hormone type 1 receptor in vascular smooth muscle cells. J Mol Cell Cardiol, 49:1012-1021.

[18] Chen J, Somanath PR, Razorenova O, et al. Akt11 regulates pathological angiogenesis, vascular maturation and permeability in vivo. Nat Med, 2005, 11:1188-1196.

[19] Alon T, Hemo I, Itin A, et al. Vascular endothelial growth factor acts as a survival factor for newly formed retinal vessels and has implications for retinopathy of prematurity. Nat Med, 1995, 1:1024-1028. 
[20]Gerber HP, McMurtrey A, Kowalski J, et al. Vascular endothelial growth factor regulates endothelial cell survival through the phosphatidylinositol 3'-kinase/Akt1 signal transduction pathway. Requirement for Flk-1/KDR activation. J Biol Chem, 1998, 273:30336-30343.

[21]Fujio Y, Walsh K. Akt1 mediates cytoprotection of endothelial cells by vascular endothelial growth factor in an anchorage-dependent manner. J Biol Chem, 1999, 274:16349-16354.

[22]Shiojima I, Walsh K. Role of Akt1 signaling in vascular homeostasis and angiogenesis. Circ Res, 2002, 90:1243-1250.

[23] Morales-Ruiz M, Fulton D, Sowa G, et al. Vascular endothelial growth factor-stimulated actin reorganization and migration of endothelial cells is regulated via the serine/threonine kinase Akt 1 . Circ Res ,2000, 86:892-896.

[24] Brazil DP, Yang ZZ, Hemmings BA. Advances in protein kinase B signalling: AKTion on multiple fronts. Trends Biochem Sci ,2004, 29:233-242.

[25] Raffetto JD, Khalil RA. Matrix metalloproteinases and their inhibitors in vascular remodeling and vascular disease. Biochem Pharmacol, 2008, 75:346-359.

[26] Rundhaug JE. Matrix metalloproteinases and angiogenesis. J Cell Mol Med ,2005, 9:267-285.

[27] Fingleton B. Matrix metalloproteinases: roles in cancer and metastasis. Front Biosci, 2006, $11: 479-491$.

[28] Opdenakker G, Van den Steen PE, Van Damme J. Gelatinase B: a tuner and amplifier of immune functions. Trends Immunol, 2001, 22:571-579.

[29] Ma DH, Chen JK, Kim WS, et al. Expression of matrix metalloproteinases 2 and 9 and tissue inhibitors of metalloproteinase 1 and 2 in inflammation-induced corneal neovascularization. Ophthalmic Res, 2001, 33:353-362.

[30] Theisen CS, Wahl JK, Johnson KR, et al. NHERF links the N-cadherin/catenin complex to the platelet-derived growth factor receptor to modulate the actin cytoskeleton and regulate cell motility. Mol Biol Cell, 2007, 18:1220-1232.

[31]Janmey PA. The cytoskeleton and cell signaling: component localization and mechanical coupling. Physiol Rev, 1998, 78:763-781.

[32] Wheeler D, Sneddon WB, Wang B, et al. NHERF-1 and the cytoskeleton regulate the traffic and membrane dynamics of G protein-coupled receptors. J Biol Chem, 2007, 282:25076-25087.

[33]Huang ZJ, Haugland RP, You WM. Phallotoxin and actin binding assay by fluorescence enhancement. Anal Biochem, 1992, 200:199-204. 